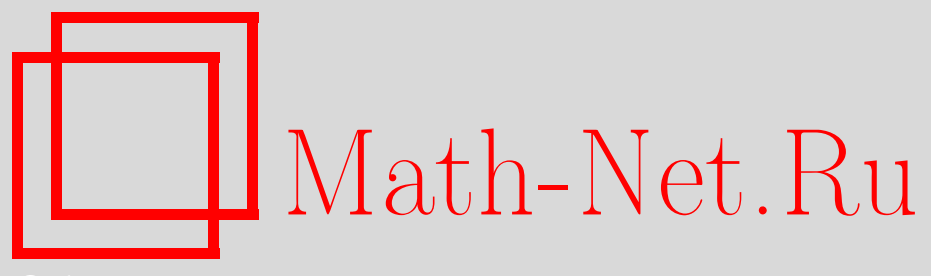

Д. В. Захаров, Изопериодические деформации акустического оператора и периодические решения уравнения Гарри Дима, ТMФ, 2007, том 153, номер 1, 46-57

DOI: https://doi.org/10.4213/tmf6120

Использование Общероссийского математического портала Math-Net.Ru подразумевает, что вы прочитали и согласны с пользовательским соглашением http: //www . mathnet.ru/rus/agreement

Параметры загрузки:

IP : 54.164 .48 .24

26 апреля 2023 г., 10:31:10

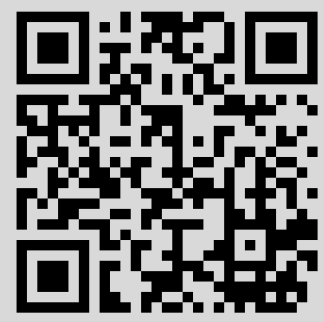




\title{
ИЗОПЕРИОДИЧЕСКИЕ ДЕФОРМАЦИИ АКУСТИЧЕСКОГО ОПЕРАТОРА И ПЕРИОДИЧЕСКИЕ РЕШЕНИЯ УРАВНЕНИЯ ГАРРИ ДИМА
}

\begin{abstract}
Рассматривается задача описания возможных спектров периодического конечнозонного акустического оператора. На пространстве модулей алгебраических римановых поверхностей построены потоки, сохраняющие периоды соответствующего оператора. С помощью подходящего расширения фазового пространства эти потоки могут быть записаны с квадратичными иррациональностями.
\end{abstract}

Ключевые слова: изопериодические деформации, периодический акустический оператор, конечнозонные решения, интегрируемые системы.

\section{1. ВВЕДЕНИЕ}

При изучении периодических потенциалов одномерного оператора Шредингера возникает следующая проблема. Периодический потенциал $u(x)$ оператора Шредингера

$$
L_{\mathrm{S}}=-\frac{d^{2}}{d y^{2}}+u(y)
$$

определяет риманову поверхность, называемую спектральной кривой. Эта кривая является алгебраической, если у потенциала конечное число запрещенных энергетических зон. С другой стороны, гиперэллиптическая кривая рода $g$ определяет потенциал $u(x)$ с соответствующим спектром, который можно продолжить до решения $u(x, \vec{t})$ иерархии Кортевега-де Фриза (КдФ). Однако в общем случае этот потенциал не является периодическим. Напрямую продолжить спектральное преобразование на квазипериодические потенциалы невозможно, например спектр квазипериодического потенциала может иметь структуру канторова множества (см. [1]), которому невозможно поставить в соответствие спектральную кривую. Задаче о выделении подмножества спектральных кривых, соответствующих строго периодическим потенциалам, в течение долгого времени не уделялось должного внимания.

\footnotetext{
*Columbia University, New York, USA. E-mail: zakharov@math.columbia.edu
} 
Периоды потенциала оператора Шредингера выражаются через абелевы интегралы на соответствующей спектральной кривой. Потенциал имеет определенный период тогда и только тогда, когда эти интегралы являются целочисленными кратными этого периода. Следовательно, подмножество кривых, соответствующих потенциалам данного периода, образуют трансцендентное подмногообразие в пространстве модулей гиперэллиптических римановых поверхностей.

Описание возможных спектров периодического оператора Шредингера (не обязательно конечнозонного) в терминах конформного отображения определенного вида было получено в работе [2]. K сожалению, этот подход является неэффективным с вычислительной точки зрения. Эффективное решение, называемое методом изопериодических деформаций, было построено в работе [3]. Это решение использует идеи, возникшие в работах [4], [5], и состоит в нахождении набора дифференциальных уравнений на спектральные данные, который не изменяет периоды решения. Эти потоки сохраняют подмногообразие кривых, соответствующих определенному периоду. После подходящего расширения фазового пространства уравнения изопериодической деформации записываются с рациональной правой частью, что делает их удобными для численных экспериментов.

В данной работе этот подход применяется для изучения периодических конечнозонных плотностей акустического оператора

$$
L_{\mathrm{A}}=-r^{2}(x) \frac{d^{2}}{d x^{2}} .
$$

Конечнозонные плотности акустического оператора и соответствующие периодические решения уравнения Гарри Дима

$$
r_{t}=r^{3} r_{x x x}
$$

были впервые построены в работах [6]-[8] с использованием преобразования Хопфа для оператора Шредингера, введенного в работе [9]. Периодические решения уравнения Гарри Дима недавно рассматривались в связи с задачей Саффмана-Тейлора (см. [10]), тогда как периодические конечнозонные плотности акустического оператора связаны с геодезическими кривыми на эллипсоиде (см. [11]-[14]).

В разделах 2, 3 приводится краткий обзор спектральной теории оператора Шредингера и метода изопериодических деформаций. В разделе 4 строится спектральная теория акустического оператора при помощи его связи с оператором Шредингера, изопериодические деформации акустического оператора получены в разделе 5 . Главным результатом данной работы является теорема 2, в которой дается явный вид уравнений деформации.

\section{2. ПЕРИОДИЧЕСКИЕ КОНЕЧНОЗОННЫЕ ПОТЕНЦИАЛЫ ОПЕРАТОРА ШРЕДИНГЕРА}

Сначала напомним спектральную теорию одномерного периодического конечнозонного оператора Шредингера (1.1), где $u(y)$ - гладкий периодический вещественный потенциал с периодом П (см., например, [15]). 
Для оператора $L_{\mathrm{S}}$ рассматриваются две спектральные задачи:

а) стандартная задача в $L^{2}(\mathbb{R})$

$$
L_{\mathrm{S}} \varphi=\lambda \varphi, \quad|\varphi(y)|<\infty, \quad y \rightarrow \pm \infty ;
$$

б) задача Дирихле на периоде

$$
L_{\mathrm{S}} \varphi=\lambda \varphi, \quad \varphi\left(y_{0}\right)=\varphi\left(y_{0}+\Pi\right)=0 .
$$

Спектр первой задачи непрерывен и состоит из бесконечного числа разрешенных зон $\left[\Lambda_{0}, \Lambda_{1}\right],\left[\Lambda_{2}, \Lambda_{3}\right], \ldots$, где $\Lambda_{2 j}<\Lambda_{2 j+1} \leqslant \Lambda_{2 j+2}$. Промежутки между этими зонами $\left(\Lambda_{2 j+1}, \Lambda_{2 j+2}\right)$, которые могут иметь нулевую длину, называются запрещенными зонами. Спектр второй задачи $d_{j}\left(y_{0}\right)$ полностью дискретен, с одним собственным значением в каждой запрещенной зоне, $d_{j}\left(y_{0}\right) \in\left[\Lambda_{2 j-1}, \Lambda_{2 j}\right]$, включая тривиальные.

Основной интерес представляет случай, когда потенциал $u(y)$ имеет конечное число запрещенных зон. Такой потенциал называется конечнозонным. Пусть $\left(-\infty, \lambda_{0}\right)$, $\left(\lambda_{1}, \lambda_{2}\right), \ldots,\left(\lambda_{2 g-1}, \lambda_{2 g}\right)$ - нетривиальные зоны, и пусть $\gamma_{j}\left(y_{0}\right)$ - собственное число задачи Дирихле, лежащее в $j$-й запрещенной зоне.

Прямое спектральное преобразование ставит в соответствие каждому конечнозонному потенциалу $u(y)$ следующий набор данных:

а) гиперэллиптическую риманову поверхность $Г$ рода $g$, оснащенную двулистным накрытием $\lambda: \Gamma \rightarrow \mathbb{C P}^{1}$ с точками ветвления $\lambda_{0}, \ldots, \lambda_{2 g}$ и $\infty$;

б) мероморфную функцию $\varphi\left(y, y_{0}, P\right)$ на поверхности $\Gamma \backslash \lambda^{-1}(\infty)$, называемую блоховской, у которой имеются $g$ простых полюсов $P_{1}\left(y_{0}\right), \ldots, P_{g}\left(y_{0}\right)$ на $\Gamma$, удовлетворяющих $\lambda\left(P_{k}\left(y_{0}\right)\right)=\gamma_{k}\left(y_{0}\right)$.

Эта функция является общей собственной функцией для оператора Шредингера и оператора монодромии:

$$
\begin{aligned}
L_{\mathrm{S}} \varphi\left(y, y_{0}, P\right) & =\lambda(P) \varphi\left(y, y_{0}, P\right), \\
\varphi\left(y+\Pi, y_{0}, P\right) & =\mu(P) \varphi\left(y, y_{0}, P\right),
\end{aligned}
$$

и имеет следующую асимптотику:

$$
\varphi\left(y, y_{0}, P\right)=e^{i\left(y-y_{0}\right) \sqrt{\lambda}}(1+o(1)) .
$$

Логарифмическая производная блоховской функции имеет вид

$$
\chi(y, P)=-i \frac{\varphi_{y}\left(y, y_{0}, P\right)}{\varphi\left(y, y_{0}, P\right)}=\frac{\sqrt{R(\lambda(P))}}{S(y, \lambda(P))}-\frac{i}{2} \frac{S_{y}(y, \lambda(P))}{S(y, \lambda(P))},
$$

где функции $R(\lambda)$ и $S(y, \lambda)$ определяются как

$$
R(\lambda)=\prod_{j=0}^{2 g}\left(\lambda-\lambda_{j}\right), \quad S(y, \lambda)=\prod_{k=1}^{g}\left(\lambda-\gamma_{k}(y)\right) .
$$

Многозначная функция $p(P)=-i \ln \mu(P) / \Pi$ называется квазиимпульсом. Ее дифференциал

$$
d p=-\frac{i}{\Pi} \frac{d \mu}{\mu}
$$


является мероморфной 1-формой на поверхности Г, он однозначно определяется следующими условиями:

1) $d p$ имеет единственный двойной полюс на бесконечности с главной частью

$$
d p=\left(-\frac{1}{w^{2}}+O(1)\right) d w
$$

где $w=\lambda^{-1 / 2}-$ локальный параметр;

2) периоды $d p$ вдоль $a$-циклов равны нулю:

$$
\oint_{a_{k}} d p=0, \quad k=1,2, \ldots, g .
$$

Так как функция $\mu(\lambda)=e^{i \Pi p(\lambda)}$ является однозначной, то $b$-периоды $d p$ являются целочисленными кратными $2 \pi / \Pi$ :

$$
\oint_{b_{k}} d p=\frac{2 \pi n_{k}}{\Pi}, \quad n_{k} \in \mathbb{Z}, \quad k=1,2, \ldots, g .
$$

$\mathrm{C}$ другой стороны, по гиперэллипической римановой поверхности $\Gamma$ и двулистному накрытию $\lambda: \Gamma \rightarrow \mathbb{C P}^{1}$ с вещественными точками ветвления $\lambda_{0}, \ldots, \lambda_{2 g}$ и $\infty$ и по неспециальному дивизору $D=P_{1}+\cdots+P_{g}$, удовлетворяющему условию $\lambda\left(P_{j}\right) \in\left[\lambda_{2 j-1}, \lambda_{2 j}\right]$, можно построить гладкий вещественный потенциал $u(y)$ оператора Шредингера со спектральными данными $\left\{\lambda_{j}, P_{k}(0)\right\}$. Этот потенциал выражается через тета-функцию Г при помощи формулы Матвеева-Итса:

$$
u(y)=-2 \partial_{y}^{2} \ln \theta\left(y \vec{U}_{1}-\vec{A}\left(P_{1}\right)-\cdots-\vec{A}\left(P_{g}\right)-\vec{K} \mid B\right)+C(\Gamma),
$$

где $\vec{A}$ - отображение Абеля, $\vec{K}$ - вектор римановых констант, $C(\Gamma)$ - постоянная, а вектор $\vec{U}_{1}$ является вектором $b$-периодов единственного мероморфного дифференциала $d p$ на $\Gamma$, удовлетворяющего условиям 1 и 2 :

$$
\left(\vec{U}_{1}\right)_{k}=\frac{1}{2 \pi} \oint_{b_{k}} d p, \quad k=1,2, \ldots, g .
$$

Этот потенциал периодический с периодом П, если компоненты вектора $\vec{U}_{1}$ являются целочисленными кратными $1 /$ П. Однако для произвольного потенциала компоненты $\vec{U}_{1}$ являются произвольными вещественными числами, поэтому потенциал $u(y)$ является, вообще говоря, квазипериодическим.

Следовательно, задача описания конечнозонных потенциалов с периодом П сводится к следующему: описать все гиперэллиптические римановы поверхности, на которых 1-форма $d p$, однозначно определяемая условиями 1 и 2 , имеет $b$-периоды, кратные $2 \pi / П$. Эффективное решение этой проблемы было дано в работе [3]. Это решение кратко описывается в следующем разделе.

\section{3. ИЗОПЕРИОДИЧЕСКИЕ ДЕФОРМАЦИИ ОПЕРАТОРА ШРЕДИНГЕРА}

Пусть $\Gamma_{0}$ - гиперэллиптическая риманова поверхность, соответствующая вещественному оператору Шредингера с периодом единица. Рассмотрим деформацию 
$\Gamma(t)$ поверхности $\Gamma_{0}$, т.е. рассмотрим непрерывное однопараметрическое семейство гиперэллиптических римановых поверхностей рода $g$, оснащенных двулистными накрытиями $\lambda(t): \Gamma(t) \rightarrow \mathbb{C P}^{1}$ с точками ветвления $\lambda_{0}(t), \ldots, \lambda_{2 g}(t)$ на вещественной оси, а также в точке $\infty$, с начальным условием $\Gamma(0)=\Gamma_{0}$. Если каждая поверхность $\Gamma(t)$ также соответствует потенциалу с периодом единица, то такая деформация называется изопериодической. Главный результат работы [3] состоит в эффективном описании всех таких деформаций.

Предположим, что $\Gamma(t)$ - изопериодическая деформация. Тогда на каждой римановой поверхности задана мероморфная 1-форма $d p(t)$, удовлетворяющая свойствам 1 и 2 и имеющая целые $b$-периоды. Рассмотрим мероморфную 1-форму

$$
\omega=\frac{\partial p}{\partial t} d \lambda-\frac{\partial \lambda}{\partial t} d p
$$

на поверхности $\Gamma_{0}$. Эта форма имеет двойной полюс на бесконечности и не имеет других особенностей, такие формы называются слабосингулярными. Если выбрать связность $\partial p / \partial t=0$, то деформация точек ветвления имеет явный вид

$$
\frac{\partial \lambda_{j}}{\partial t}=-\frac{\omega\left(\lambda_{j}\right)}{d p\left(\lambda_{j}\right)} .
$$

Наоборот, по слабосингулярной 1-форме $\omega$ можно построить деформацию поверхности $\Gamma_{0}$, используя эту формулу. Если теперь выбрать связность в виде $\partial \lambda / \partial t=0$, то мы получим, что функция

$$
\frac{\partial p}{\partial t}=\frac{\omega}{d \lambda}
$$

является однозначной функцией на поверхности $\Gamma_{0}$. Следовательно, периоды $d p(t)$ остаются постоянными, и если $\Gamma_{0}$ соответствует, в смысле прямого спектрального преобразования, потенциалу с периодом единица, тогда то же самое можно сказать и про все поверхности $\Gamma(t)$. Следовательно, изопериодические деформации оператора Шредингера описываются мероморфными 1-формами с особенностями определенного вида. Этот подход используется для построения изопериодических деформаций акустического оператора.

\section{4. СПЕКТРАЛЬНАЯ ТЕОРИЯ АКУСТИЧЕСКОГО ОПЕРАТОРА}

Рассмотрим теперь акустический оператор (1.2), где функция $r(x)$, называемая плотностъю, является гладкой и положительной. Спектральная теория акустического оператора широко изучена. Спектр задачи

$$
L_{\mathrm{A}} \psi(x, \lambda)=\lambda \psi(x, \lambda)
$$

в пространстве $L^{2}(\mathbb{R})$, как и спектр оператора Шредингера, имеет зонную структуру и состоит из бесконечного числа разрешенных зон $\left[\Lambda_{0}, \Lambda_{1}\right],\left[\Lambda_{2}, \Lambda_{3}\right], \ldots$, где $\Lambda_{2 j}<\Lambda_{2 j+1} \leqslant \Lambda_{2 j+2}$. Если плотность $r(x)$ является гладкой, то имеется дополнительное условие $\Lambda_{0}=0$. Конечнозонные плотности оператора $L_{\mathrm{A}}$ можно построить с помощью следующего известного предложения [9]. 
ПреДЛОЖЕНИЕ. Предположим, что функиии $r(x), \psi(x, \lambda)$ удовлетворяют акустическому уравнению

$$
-r^{2}(x) \frac{d^{2}}{d x^{2}} \psi(x, \lambda)=\lambda \psi(x, \lambda)
$$

Въполним замену переменных

$$
y(x)=\int_{0}^{x} \frac{d x^{\prime}}{r\left(x^{\prime}\right)}
$$

Тогда функиии

$$
\begin{gathered}
u(y)=\frac{1}{4} r_{x}^{2}(x)-\frac{1}{2} r(x) r_{x x}(x), \\
\varphi(y, \lambda)=\psi(x, \lambda) r^{-1 / 2}(x)
\end{gathered}
$$

удовлетворяют уравнению Шредингера

$$
-\frac{d^{2}}{d y^{2}} \varphi(y, \lambda)+u(y) \varphi(y, \lambda)=\lambda \varphi(y, \lambda) .
$$

Если $r(x)$ - периодическая плотность оператора $L_{\mathrm{A}}$ с периодом $T$, то и $(y)$ - периодический потенииал $L_{\mathrm{S}}$ с периодом

$$
\Pi=\int_{x_{0}}^{x_{0}+T} \frac{d x}{r^{\prime}(x)},
$$

и операторы $L_{\mathrm{A}}$ и $L_{\mathrm{S}}$ имеют одинаковый спектр в $L^{2}(\mathbb{R})$.

Это предложение позволяет нам строить конечнозонную плотность акустического оператора по периодическому потенциалу $u(y)$ оператора Шредингера, заданному формулой Матвеева-Итса для какого-либо набора спектральных данных $\left\{\lambda_{i}, \gamma_{k}\right\}$ с $\lambda_{0}=0$, решая обратную задачу для уравнений (4.3), (4.4). Обратное преобразование определено только с точностью до параметра, соответствующего калибровочной свободе акустического уравнения:

$$
x \rightarrow \alpha x, \quad r(x) \rightarrow \alpha^{-1} r(\alpha x) .
$$

Таким образом, периодическому потенциалу соответствует однопараметрическое семейство периодических плотностей. Параметр $\alpha$ должен рассматриваться как дополнительный параметр задачи.

Явное выражение для $r(x)$ через тета-функции было получено в работах [6]-[8] продолжением преобразования Хопфа (4.3)-(4.5) до преобразования между иерархиями Гарри Дима и КдФ. Таким образом, конечнозонные периодические плотности акустического оператора эквивалентны $x$-периодическим решениям уравнения Гарри Дима (1.3).

Так как периодические плотности соответствуют периодическим потенциалам, а последние описываются изопериодическими деформациями, то задача описания 
всех периодических плотностей акустического оператора в известном смысле решена. Однако хотелось бы найти естественный выбор калибровочной константы, явно зависящий от спектральных данных, а также набор дифференциальных уравнений на спектральные данные акустического оператора, чтобы период единственной плотности, соответствующей спектральным данным, сохранялся бы этими потоками. Обратимся теперь к этой проблеме.

Пусть $r(x)$ - конечнозонная плотность с периодом $T$, и пусть $\psi_{ \pm}\left(x, x_{0}, \lambda\right)$ - блоховская функция соответствующего акустического оператора $L_{\mathrm{A}}$, т.е. общая собственная функция акустического оператора и оператора монодромии,

$$
\begin{aligned}
L_{\mathrm{A}} \psi_{ \pm}\left(x, x_{0}, \lambda\right) & =\lambda \psi_{ \pm}\left(x, x_{0}, \lambda\right), \\
\psi_{ \pm}\left(x+T, x_{0}, \lambda\right) & =e^{ \pm i T q(\lambda)} \psi_{ \pm}\left(x, x_{0}, \lambda\right),
\end{aligned}
$$

нормированная условием

$$
\left.\psi_{ \pm}\left(x, x_{0}, \lambda\right)\right|_{x=x_{0}}=1 .
$$

Пусть $L_{\mathrm{S}}$ - соответствующий оператор Шредингера, заданный уравнениями (4.3)-(4.5). Тогда функцию $\psi\left(x, x_{0}, \lambda\right)$ можно выразить через блоховскую функцию (2.1) оператора $L_{\mathrm{S}}$ следующим образом:

$$
\psi_{ \pm}\left(x, x_{0}, \lambda\right)=r^{-1 / 2}\left(x_{0}\right) r^{1 / 2}(x) \varphi\left(y, y_{0}, \lambda(P)\right) .
$$

Таким образом, можно рассматривать блоховскую функцию как мероморфную функцию на спектральной кривой $Г$ оператора $L_{\mathrm{S}}$, т.е. можно положить

$$
\psi_{ \pm}\left(x, x_{0}, \lambda\right)=\psi\left(x, x_{0}, \lambda(P)\right),
$$

где $\psi\left(x, x_{0}, P\right)$ - мероморфная функция на $\Gamma$, удовлетворяющая уравнениям

$$
\begin{aligned}
L_{\mathrm{A}} \psi\left(x, x_{0}, P\right) & =\lambda(P) \psi\left(x, x_{0}, P\right), \\
\psi\left(x+T, x_{0}, P\right) & =e^{i T q(P)} \psi\left(x, x_{0}, P\right),
\end{aligned}
$$

где многозначная функция $q(P)$ называется квазиимпульсом акустического оператора $L_{\mathrm{A}}$. Ее можно выразить через логарифмическую производную блоховской функции

$$
\xi(x, P)=-i \frac{\psi_{x}\left(x, x_{0}, P\right)}{\psi\left(x, x_{0}, P\right)}
$$

следующим образом:

$$
q(P)=\frac{1}{T} \int_{x_{0}}^{x_{0}+T} \xi(x, P) d x .
$$

Заметим, что функцию $\xi(x, P)$ можно выразить через логарифмическую производную $\chi(y, P)$ функции $\varphi\left(y, y_{0}, P\right)$ следующим образом:

$$
\xi(x, P)=\frac{1}{r(x)} \chi(y, P)-\frac{i}{2} \frac{r_{x}(x)}{r(x)} .
$$


С учетом (2.3) получим

$$
\xi(x, P)=\frac{\sqrt{R(\lambda)}}{r(x) S(x, \lambda)}-\frac{i}{2} \frac{r^{\prime}(x)}{r(x)}-\frac{i}{2} \frac{S_{x}(x, \lambda)}{S(x, \lambda)},
$$

где

$$
R(\lambda)=\lambda \prod_{j=1}^{2 g}\left(\lambda-\lambda_{j}\right), \quad S(x, \lambda)=\prod_{k=1}^{g}\left(\lambda-\gamma_{k}(y(x))\right) .
$$

Следовательно, квазиимпульс $q(P)$ и его дифференциал имеют следующую высокоэнергетическую асимптотику:

$$
\begin{aligned}
q(P) & =\frac{1}{w T} \int_{x_{0}}^{x_{0}+T} \frac{d x}{r(x)}+O(1), \\
d q(P) & =\left(-\frac{1}{w^{2} T} \int_{x_{0}}^{x_{0}+T} \frac{d x}{r(x)}+O(1)\right) d w
\end{aligned}
$$

где $w=\lambda^{-1 / 2}$.

Несложное вычисление показывает, что функция $\xi(x, P)$ удовлетворяет уравнению Риккати:

$$
-i \xi^{\prime}(x, P)+\xi^{2}(x, P)-\frac{\lambda(P)}{r^{2}(x)}=0 .
$$

Спектр акустического оператора всегда начинается с $\lambda=0$, т.е. наименьшей точкой ветвления Г всегда является точка $\lambda=0$. Рассмотрим уравнение Риккати вблизи этой точки. Выберем $z=\sqrt{\lambda}$ в качестве локального параметра и рассмотрим ряд Тейлора $\xi(x, P)$ в точке $z=0$ :

$$
\xi(x, P)=\xi_{0}(x)+\xi_{1}(x) z+\xi_{2}(x) z^{2}+O\left(z^{3}\right) .
$$

Подставив это выражение в уравнение Риккати, получим, что $\xi_{0}(x)=0, \xi_{1}(x)=C$, где $C$ - константа, и что $\xi_{2}(x)$ является полной производной. Следовательно, в точке $z=0$ квазиимпульс $q(P)$ и его дифференциал имеют вид

$$
q(P)=C z+O\left(z^{3}\right), \quad d q(P)=\left(C+O\left(z^{2}\right)\right) d z .
$$

С другой стороны, сравнив полученное выражение с (4.16), мы имеем

$$
C=\frac{\sqrt{\lambda_{1} \ldots \lambda_{2 g}}}{(-1)^{g} r(x) \gamma_{1}(x) \ldots \gamma_{g}(x)},
$$

и, следовательно, $r(x)$ выражается через спектральные данные и дополнительную константу $C$ следующим образом:

$$
r(x)=\frac{\sqrt{\lambda_{1} \ldots \lambda_{2 g}}}{(-1)^{g} C \gamma_{1}(x) \ldots \gamma_{g}(x)} .
$$

Константа $C$ соответствует калибровочному преобразованию (4.7) и должна рассматриваться как дополнительный, наряду с $\lambda_{j}$ и $\gamma_{k}$, спектральный параметр задачи. 
Для построения изопериодических деформаций нам потребуется правило выбора константы $C$. Естественным представляется выбор $C=(-1)^{g}$. При этом выборе значение $d q$ в точке $\lambda=0$ не зависит от спектральной кривой $\Gamma$, что будет использовано при построении изопериодических деформаций. Кроме того, при этом выборе константы $C$ бесконечно малый потенциал $u(x) \rightarrow 0$ или, что то же самое, бесконечно малые энергетические зоны $\left(\lambda_{2 j-1}-\lambda_{2 j} \rightarrow 0\right)$ соответствуют плотности $r(x) \rightarrow 1$, нормированной на единицу.

Основные результаты этого раздела можно сформулировать в следующей теореме.

Теорема 1. Пусть $\left\{\lambda_{i}, \gamma_{k}(y)\right\}$ - спектральные данные конечнозонного периодического оператора Шредингера $L_{\mathrm{S}}$ с потенииалом $u(y)$, причем $\lambda_{0}=0$. Тогда плотность $r(x)$ акустического оператора $L_{\mathrm{A}}$, связанного с $u(y)$ соотношениями (4.3)-(4.5), задается равенством (4.23), где $C$ - произволъная постоянная. При выборе $C=(-1)^{g}$ дифберенииал квазиимпульса $L_{\mathrm{A}}$ имеет полюс второго порядка в точке $\lambda=\infty$ с главной частью (4.18) и фиксированные члены первой и второй степени в точке $\lambda=0$ :

$$
d q(P)=\left((-1)^{g}+O\left(z^{2}\right)\right) d z .
$$

Используя эту теорему, можно построить изопериодические деформации акустического оператора.

\section{5. ИЗОПЕРИОДИЧЕСКИЕ ДЕФОРМАЦИИ АКУСТИЧЕСКОГО ОПЕРАТОРА}

Напомним, что деформация римановой поверхности задается мероморфной 1-формой

$$
\omega=\frac{\partial q}{\partial t} d \lambda
$$

Чтобы задавать изопериодическую деформацию акустического оператора, эта форма должна удовлетворять следующим требованиям.

1. Дифференциал квазиимпульса $d q$ имеет двойной полюс на бесконечности, следовательно, $\partial q / \partial t$ имеет там простой полюс. Так как $d \lambda$ имеет на бесконечности полюс третьего порядка, то у $\omega$ на бесконечности должен быть полюс четвертого порядка.

2. У дифференциала $d q$ в нуле фиксированы члены первого и второго порядка, следовательно, $\partial q / \partial t$ имеет нуль третьего порядка (при нашем выборе константы $C$ ). Так как у $d \lambda$ имеется простой нуль, то у $\omega$ - нуль четвертого порядка в точке $\lambda=0$.

Таким образом, 1-форма $\omega$ задает изопериодическую деформацию тогда и только тогда, когда она имеет дивизор $4 \cdot 0-4 \cdot \infty$. Пространство таких форм $g$-мерно. Так как пространство спектральных кривых имеет размерность $2 g$, а кривые, соответствующие плотностям с периодом единица, выделяются $g$ условиями, то формы такого вида образуют базис изопериодических деформаций.

Как и ранее, деформация задается в явном виде через точки ветвления:

$$
\frac{\partial \lambda_{j}}{\partial t}=-\frac{\omega\left(\lambda_{j}\right)}{d q\left(\lambda_{j}\right)}, \quad j=1,2, \ldots, 2 g .
$$


На гиперэллиптической поверхности 1-форму $d q$ можно явно записать как

$$
d q=\frac{Q(\lambda)}{2 \sqrt{R(\lambda)}} d \lambda,
$$

где $Q(\lambda)=q_{g} \lambda^{g}+\cdots+q_{0}, q_{0}=(-1)^{g} \sqrt{\lambda_{1} \ldots \lambda_{2 g}}$. Коэффициенты многочлена $Q(\lambda)$ определяются обращением в нуль $a$-периодов $d q$ и выражаются через гиперэллиптические интегралы.

Произвольную 1-форму $\omega$ с дивизором $4 \cdot 0-4 \cdot \infty$ можно записать как

$$
\omega=\frac{f(\lambda)}{2 \sqrt{R(\lambda)}} d \lambda,
$$

где

$$
f(\lambda)=f_{g+1} \lambda^{g+1}+\cdots+f_{2} \lambda^{2}=\lambda^{2}\left(f_{g+1} \lambda^{g-1}+\cdots+f_{2}\right) .
$$

Деформация, задаваемая такой формой, имеет вид

$$
\frac{\partial \lambda_{j}}{\partial t}=-\frac{f\left(\lambda_{j}\right)}{Q\left(\lambda_{j}\right)}, \quad j=1,2, \ldots, 2 g
$$

Можно было бы выбрать базис в пространстве мероморфных 1-форм с дивизором $4 \cdot 0-4 \cdot \infty$ и построить по этой формуле базис изопериодических деформаций. Однако правая часть уравнений деформации содержит коэффициенты многочлена $Q(\lambda)$, которые, в свою очередь, выражаются через гиперэллиптические интегралы, содержащие точки ветвления. Чтобы избежать этой трудности, воспользуемся подходом из работы [3]. Разложим многочлен $Q(\lambda)$ на множители,

$$
Q(\lambda)=\left(\beta_{1} \lambda-\sqrt{\lambda_{1} \lambda_{2}}\right) \ldots\left(\beta_{g} \lambda-\sqrt{\lambda_{2 g-1} \lambda_{2 g}}\right),
$$

и будем рассматривать величины $\beta_{k}$ как независимые параметры, которые деформируются вместе с точками ветвления. Такое расширение фазового пространства значительно упрощает уравнения деформации.

Нетрудно показать, что деформации $\beta_{k}$ задаются следующей леммой.

Лемма 1. Пусть $\partial / \partial t-$ поток, заданный формой $\omega$ с многочленом $f(\lambda)$. Тогда дебормации $\lambda_{j}$ и $\beta_{k}$ имеют вид

$$
\begin{gathered}
\frac{\partial \lambda_{j}}{\partial t}=-\frac{f\left(\lambda_{j}\right)}{Q\left(\lambda_{j}\right)}, \quad j=1,2, \ldots, 2 g \\
\frac{\partial \beta_{k}}{\partial t}=\beta_{k}\left[-\frac{1}{2 \lambda_{2 k-1}} \frac{f\left(\lambda_{2 k-1}\right)}{Q\left(\lambda_{2 k-1}\right)}-\frac{1}{2 \lambda_{2 k}} \frac{f\left(\lambda_{2 k}\right)}{Q\left(\lambda_{2 k}\right)}+\right. \\
+\left.\frac{1}{Q^{\prime}(\lambda)}\left(f^{\prime}(\lambda)-\frac{f(\lambda) R^{\prime}(\lambda)}{2 R(\lambda)}\right)\right|_{\lambda=\frac{\sqrt{\lambda_{2 k-1} \lambda_{2 k}}}{\beta_{k}}}
\end{gathered}
$$

Как мы видим, уравнения деформации более не содержат гиперэллиптических интегралов, что делает их удобными для численных экспериментов. 
Чтобы получить явный вид уравнений деформации, выберем базис в пространстве дифференциалов с дивизором $4 \cdot 0-4 \cdot \infty$ следующим образом:

$$
\omega_{k}=\frac{c_{k} \lambda^{2}}{\beta_{k} \lambda-\sqrt{\lambda_{2 k-1} \lambda_{2 k}}} d q, \quad k=1,2, \ldots, g,
$$

где $c_{k}-$ произвольные константы. Тогда

$$
\frac{f_{k}(\lambda)}{Q(\lambda)}=\frac{c_{k} \lambda^{2}}{\beta_{k} \lambda-\sqrt{\lambda_{2 k-1} \lambda_{2 k}}}, \quad k=1,2, \ldots, g,
$$

и уравнения деформации приводятся в следующей теореме.

ТеОРема 2. Пусть Г - гиперэллиптическая кривая, соответствующая гладкой плотности с периодом единица и с точками ветвления $\lambda_{0}=0, \lambda_{1}, \ldots, \lambda_{2 g}$. Пусть $\sqrt{\lambda_{2 k-1} \lambda_{2 k}} / \beta_{k}, k=1,2, \ldots, g,-$ нули квазиимпульса. Рассмотрим потоки

$$
\begin{gathered}
\frac{\partial \lambda_{j}}{\partial t_{k}}=-\frac{c_{k} \lambda_{j}^{2}}{\beta_{k} \lambda_{j}-\sqrt{\lambda_{2 k-1} \lambda_{2 k}}}, \quad j=1,2, \ldots, 2 g, \quad k=1,2, \ldots, g, \\
\frac{\partial \beta_{l}}{\partial t_{k}}=c_{k} \beta_{k}\left[-\frac{1}{2 \lambda_{2 l-1}} \frac{\lambda_{2 l-1}^{2}}{\beta_{k} \lambda_{2 l-1}-\sqrt{\lambda_{2 k-1} \lambda_{2 k}}}-\frac{1}{2 \lambda_{2 l}} \frac{\lambda_{2 l}^{2}}{\beta_{k} \lambda_{2 l}-\sqrt{\lambda_{2 k-1} \lambda_{2 k}}}+\right. \\
\left.+\frac{\lambda_{2 l-1} \lambda_{2 l}}{\beta_{l}^{2}}\left(\beta_{k} \frac{\sqrt{\lambda_{2 l-1} \lambda_{2 l}}}{\beta_{l}}-\sqrt{\lambda_{2 k-1} \lambda_{2 k}}\right)^{-1}\right], \quad l=1,2, \ldots, g, \quad l \neq k, \\
\frac{\partial \beta_{k}}{\partial t_{k}}=c_{k} \beta_{k}\left\{-\frac{1}{2 \lambda_{2 k-1}} \frac{\lambda_{2 k-1}^{2}}{\beta_{k} \lambda_{2 k-1}-\sqrt{\lambda_{2 k-1} \lambda_{2 k}}}-\frac{1}{2 \lambda_{2 l}} \frac{\lambda_{2 k}^{2}}{\beta_{k} \lambda_{2 k}-\sqrt{\lambda_{2 k-1} \lambda_{2 k}}}+{ }^{-1}+2 \frac{\sqrt{\lambda_{2 k-1} \lambda_{2 k}}}{\beta_{k}}-\right. \\
\left.\left.+\frac{1}{\beta_{k}}\left[\frac{\lambda_{2 k-1} \lambda_{2 k}}{\beta_{k}^{2}} \sum_{l \neq k} \beta_{l}\left(\beta_{l} \frac{\sqrt{\lambda_{2 k-1} \lambda_{2 k}}}{\beta_{k}}-\sqrt{\lambda_{2 l-1} \lambda_{2 l}}\right)^{-1}\right)\right]\right\}, \quad k=1,2, \ldots, g, \\
\left.\left.-\frac{\lambda_{2 k-1} \lambda_{2 k}}{\beta_{k}}\left(\frac{\beta_{k}}{\sqrt{\lambda_{2 k-1} \lambda_{2 k}}}-\sum_{j=1}^{2 g} \frac{\beta_{k}}{\sqrt{\lambda_{2 k-1} \lambda_{2 k}}-\lambda_{j} \beta_{k}}\right)\right]\right\}
\end{gathered}
$$

на спектральных данных, где $\partial / \partial t_{k}-$ поток, заданный формой $\omega_{k}$, определенной формулой (5.9). Тогда любая риманова поверхность, полученная движением вдоль этих потоков в пространстве модулей гиперэллиптических римановых поверхностей, соответствует плотности $r(x)$ акустического оператора с периодом единица. Потоки такого вида образуют базис изопериодических деформаций акустического оператора.

Мы видим, что эти расширенные уравнения не содержат гиперэллиптических интегралов.

Благодарности. Данная работа была частично выполнена во время работы автора в Национальной лаборатории в Лос-Аламосе в рамках летней программы “Математическое моделирование и анализ" в 2004 году. Автор выражает глубокую благодарность своему научному руководителю П. Г. Гриневичу. Автор благодарен И. М. Кричеверу, указавшему на ряд неточностей в первоначальном варианте работы, а также М. Степанову за многочисленные обсуждения. 


\section{Список литературы}

[1] Е. И. Динабург, Я. Г. Синай, Функи. анализ и его прилож., 9:4 (1975), 8-21.

[2] В. А. Марченко, И. В. Островский, Матем. сб., 97:4 (1975), 540-606.

[3] P. G. Grinevich, M. U. Schmidt, Phys. D, 87:1-4 (1995), 73-98.

[4] N. M. Ercolani, M. G. Forest, D. W. McLaughlin, A. Sinha, J. Nonlinear Sci., 3:1 (1993), 393-426.

[5] I. M. Krichever, Comm. Pure Appl. Math., 47:4 (1994), 437-475.

[6] L. A. Dmitrieva, J. Phys. A, 26 (1993), 6005-6020.

[7] L. A. Dmitrieva, Phys. Lett. A, 182:1 (1993), 65-70.

[8] L. A. Dmitrieva, D. A. Pyatkin, Phys. Lett. A, 303:1 (2002), 37-44.

[9] C. Rogers, M. C. Nucci, Phys. Scripta, 33:4 (1986), 289-292.

[10] S. Tanveer, Philos. Trans. Roy. Soc. London Ser. A, 343 (1993), 155-204.

[11] H. Knörrer, J. Reine Angew. Math., 334 (1982), 69-78.

[12] J. Moser, "Various aspects of integrable Hamiltonian systems", Dynamical Systems, C.I.M.E. Summer School (Bressanone, 1978), Progr. Math., 8, eds. J. Guckenheimer, J. Moser, Sh. E. Newhouse, Birkhäuser, Boston, 1980, 233-289.

[13] А.П. Веселов, Функи. анализ и его прилож., 14:1 (1980), 48-50.

[14] А.П. Веселов, Функи. анализ и его прилож., 26:3 (1992), 74-76.

[15] В. Е. Захаров, С. В. Манаков, С.П. Новиков, Л. П. Питаевский, Теория солитонов. Метод обратной задачи, Наука, М., 1980. 